\title{
Oportunidades que presenta la georreferenciación de los registros de edificios
}

\section{Opportunities presented by georeferencing of building registers}

Begoña Serrano-Lanzarote $^{(*)}$, Teresa Soto-Vicario ${ }^{(*)}$

\section{RESUMEN}

La elaboración del Informe de Evaluación del Edificio (IEE) como condición previa para solicitar financiación de obras de conservación, accesibilidad universal o eficiencia energética y las ayudas económicas a la redacción misma del IEE, han supuesto importantes estímulos para su implantación. También se ha avanzado en la regulación de la creación de organismos de registro que integren la información de los IEE, de forma que pueda ser consultada públicamente. El interés de la Administración en desarrollar herramientas informáticas que registren información sobre el estado de los edificios y además la referencien geográficamente resulta esencial para plantear políticas realistas de vivienda, rehabilitación y regeneración urbana, y además constituyen bases de datos muy útiles para llevar a cabo posteriores trabajos de investigación. En este trabajo se presentan y analizan diversos ejemplos recientes de registros y observatorios de vivienda, mostrando el potencial e interés que presentan.

Palabras clave: Registro, georreferenciación, inspección, rehabilitación, evaluación energética, observatorio.

\section{ABSTRACT}

The drafting of the Building Assessment Report (IEE) as a requirement to request funding for conservation works, universal accessibility or energy efficiency, and the financial support for the draft of the IEE itself have meant a relevant encouragement for its implementation. An improvement has also been achieved regarding the national and regional regulation on the creation of registration bodies which integrate the information contained in the IEE, in order to be publicly consulted. The interest of the Administration in developing computer tools that register and geographically reference information on the condition of buildings is essential to propose realistic policies of housing, urban rehabilitation and urban regeneration, and also constitute very useful databases to carry out subsequent research work. In this work, several recent examples of housing registers and observatories are presented and analyzed, showing the potential and interest that they present.

Keywords: Register, geographically reference, inspection, refurbishment, energy evaluation, observatory.

(*) Dra. Arquitecta, Universitat Politècnica de València, Escuela Técnica Superior de Arquitectura, Valencia (España).

(**) Arquitecta, Instituto Valenciano de la Edificación, Valencia (España)

Persona de contacto/Corresponding author: apserlan@mes.upv.es (B. Serrano-Lanzarote)

ORCID: https://orcid.org/oooo-0002-9606-0029 (B. Serrano-Lanzarote); ORCID: https://orcid.org/oooo-0003-3694-6386

(T. Soto-Vicario)

Cómo citar este artículo/Citation: Serrano-Lanzarote, Begoña.; Soto-Vicario, Teresa (2020). Oportunidades que presenta la georreferenciación de los registros de edificios. Informes de la Construcción, 72(559): e356. https://doi.org/10.3989/ic.71336.

Copyright: (C) 2020 CSIC. Este es un artículo de acceso abierto distribuido bajo los términos de la licencia de uso y distribución Creative Commons Reconocimiento 4.0 Internacional (CC BY 4.0). 


\section{INTRODUCCIÓN}

El conocimiento del estado actual del parque edificatorio en España es de gran relevancia para los distintos agentes implicados en su conservación, al ser esta realmente ineludible por la antigüedad de las construcciones. Según el Censo de Población y Vivienda de 2011 (1), aproximadamente dos terceras partes del parque residencial en España supera los $5^{0}$ años. Estos datos muestran una clara necesidad de impulsar la inspección, la rehabilitación y el registro de la información, como se ha venido reflejando en las exigencias de la normativa estatal y autonómica.

El Código Civil de 1889 (2) ya establecía la obligación de ejecutar las obras necesarias y reparar los edificios. Asimismo, las sucesivas Leyes del Suelo han ido incorporando en su articulado el deber de conservación de las edificaciones. La Ley del Suelo de 1956 (3) exigía a los propietarios el mantenimiento de los edificios en condiciones de seguridad, salubridad y ornato públicos, términos que se han mantenido en las leyes posteriores. Estas han ido añadiendo otras temáticas según evolucionaban las circunstancias. La Ley del Suelo de 1976 (4) contemplaba la figura de los Planes Especiales para la mejora del medio urbano o rural en relación al estado de conservación de las edificaciones, y obligaba a realizar las obras requeridas por planes, normas o proyectos de carácter histórico o artístico. Más adelante, la Ley del Suelo de 1990 (5), así como el texto refundido de la Ley del Suelo de 1992 (6) añadía la ejecución de obras de conservación y reforma en fachadas o espacios visibles por motivos turísticos y culturales. Seguidamente, la Ley del Suelo de 1998 (7) se limitaba a establecer el deber de conservación del mismo modo que la legislación anterior, mientras que la Ley de Ordenación de la Edificación (8) concretaba el deber de conservación de la obra ejecutada, según las instrucciones de uso y mantenimiento contenidas en el Libro del Edificio. La exigencia del deber de conservación se mantuvo en los mismos términos en la Ley del Suelo de 2007 (9), y su texto refundido de 2008 (10), ambos orientados fundamentalmente a generar nuevo crecimiento urbanístico. Sin embargo, el vigente texto refundido de la Ley del Suelo de 2015 (11), se centra en el fomento de la rehabilitación, el desarrollo sostenible y la calidad de vida de los ciudadanos, y añade a lo anterior la obligación de conservar las condiciones de accesibilidad universal y realizar obras para la mejora de la calidad y sostenibilidad del medio urbano.

Fue a partir de los años 90 cuando el deber de conservación de los edificios se hizo realmente efectivo para los propietarios con la obligación de realizar una Inspección Técnica de Edificios (ITE), regulada por las normativas urbanísticas y territoriales de las comunidades autónomas.

A nivel estatal, con el Real Decreto Ley 8/2011 (12), se empezó a exigir a nivel nacional la inspección técnica periódica en edificios de uso preferentemente residencial, de antigüedad superior a 50 años y situados en municipios con población superior a 25.00o habitantes, los cuales debían contar con una ordenanza específica. En ese mismo año, la Ley de Economía Sostenible (13) estableció la obligación de que los propietarios de edificios residenciales realizaran obras de conservación que garantizaran los derechos de personas con discapacidad, la seguridad, así como la adecuación de instalaciones y servicios mínimos, la reducción de emisiones contaminantes y del consumo de agua y energía. Con estos antece- dentes, el Plan Estatal de fomento del alquiler de viviendas, la rehabilitación edificatoria, y la regeneración y renovación urbanas, 2013-2016 (14), estableció el modelo tipo de Informe de Evaluación del Edificio (IEE) para edificios de tipología residencial colectiva, que incluía el estado de conservación e incorporaba las condiciones básicas de accesibilidad según el Código Técnico de la Edificación (15), así como el Certificado de Eficiencia Energética del Edificio (16). Partiendo de esta premisa, la Ley 8/2013 (17) estableció la regulación básica del IEE en iguales términos y exigió el informe en edificios de tipología residencial colectiva con una antigüedad superior a 50 años, así como en edificios cuyos titulares se acogieran a ayudas públicas para obras de conservación, accesibilidad universal o eficiencia energética. Un aspecto interesante de la Ley 8/2013 era que los propietarios debían remitir una copia del IEE al organismo determinado por la comunidad autónoma, con el fin de que dicha información formara parte de un registro integrado único. Sin embargo, en lo referente al IEE, la Ley 8/2013 fue declarada parcialmente inconstitucional (18). Con todos estos precedentes, el Ministerio de Fomento implantó a nivel nacional una herramienta informática para la redacción del IEE.

En el caso de la Comunitat Valenciana, y con lo establecido hasta entonces por la legislación estatal, la vigente Ley 1/2019, de 5 de febrero, de la Generalitat, de modificación de la Ley 5/2014, de 25 de julio, de ordenación del territorio, urbanismo y paisaje de la Comunitat Valenciana (19), dispone en su artículo 180 que los propietarios de toda edificación con uso residencial destinado a vivienda de antigüedad superior a cincuenta años, deberán promover, al menos cada diez años, la realización de una inspección técnica para evaluar el estado de conservación del edificio. Dicha evaluación también es preceptiva si los propietarios pretenden acogerse a ayudas públicas para acometer obras de conservación, accesibilidad universal o eficiencia energética. Mediante la Resolución del 8 de septiembre de 2014 (20), se indicaba que la incorporación del certificado de eficiencia energética a la inspección técnica representaba el procedimiento oficial IEE a nivel autonómico como ITE y como informe previo a la concesión de ayudas, pasando a denominarse IEE.CV.

En relación a la financiación, la Orden 7/2015 (21), apoyaba la implantación del IEE.CV con ayudas particulares para su redacción, y en el año siguiente la Orden 5/2016 (22), regulaba las ayudas para la rehabilitación de elementos comunes de edificios de viviendas, vinculándolas a la redacción del IEE. CV y especificaba las actuaciones subvencionables: conservación, mejora de la calidad y sostenibilidad, y accesibilidad. En definitiva, desde el año 2015 hasta la actualidad se ha venido fomentando el IEE.CV mediante financiación para su redacción y su vinculación a las ayudas económicas para la rehabilitación, lo que ha supuesto un importante impulso para su implantación.

Regresando al ámbito estatal, el vigente texto refundido de la Ley de Suelo y Rehabilitación Urbana de 2015 (23), vuelve a determinar los tipos de edificios y las fechas y plazos en los que éstos deben contar con el IEE, sin perjuicio de que las comunidades autónomas aprueben una regulación más exigente y según dispongan las ordenanzas municipales. Asimismo, esta disposición establece que la Administración General del Estado, en colaboración con las comunidades autónomas, definirá la formación y actualización permanentes de un sistema público general e integrado, con censos de edificios que 
precisen rehabilitación, a partir de la información contenida en los IEE.

Actualmente, en la Comunitat Valenciana, el vigente Decreto 53/2018 (24) regula el contenido, formalización, tramitación y registro oficial del IEE para edificios de uso residencial, que pasa a designarse IEEV.CV. Según esta última disposición, el contenido del IEEV.CV comprende el estado de conservación del edificio y las intervenciones a realizar a medio plazo o de forma urgente, así como la valoración de las condiciones básicas de accesibilidad y la eficiencia energética. Como ayuda para la elaboración del informe IEEV.CV, se ha generado un detallado procedimiento acompañado de una herramienta informática (25), para identificar y calificar las lesiones, las condiciones de accesibilidad y los datos de la envolvente térmica del edificio, que pueden ser importados por el programa informático Método Abreviado CERMA v. 4.2.5 (26). De este modo, se genera el certificado de eficiencia energética de manera automática para su envío y registro telemático con el resto del informe IEEV.CV al organismo competente de la Comunitat Valenciana. Resulta interesante lo establecido en el artículo 4 del Decreto 53/2018, referente al hecho de que los datos y resultados del IEEV.CV serán antecedentes de referencia para la confección, en su caso, del programa de mantenimiento que forma parte del Libro del Edificio rehabilitado, dado que ello constituye una novedad en el panorama normativo nacional del IEE. Para facilitar este proceso, desde la Generalitat Valenciana se ha impulsado la creación de una aplicación web (27) que importa los datos del IEEV.CV y desarrolla un programa de operaciones de mantenimiento del edificio existente. Igualmente, el mencionado Decreto 53/2018 determina la creación del registro autonómico del IEEV.CV, en el cual se inscriben de forma telemática los IEEV.CV y que es gestionado por el departamento de la Generalitat Valenciana competente en materia de vivienda.

Finalmente, en referencia al espacio urbano, el vigente Plan Estatal de Vivienda 2018-2021 (28), establece que para el reconocimiento de las ayudas previstas en el programa el área de regeneración y renovación urbana o rural, es necesaria la delimitación cartográfica del área en cuestión, además de un diagnóstico sobre su situación demográfica, social, económica y ambiental. Para realizar esta evaluación es necesario que la Administración ofrezca información actualizada y georreferenciada del estado de la zona urbana concreta.

\section{PROBLEMÁTICA ACTUAL}

Según se afirma en el preámbulo de la ya mencionada Ley 8/2013, actualmente la Inspección Técnica de Edificios (ITE) es el único instrumento que permite determinar el grado de conservación de los inmuebles. Sin embargo, este instrumento no está establecido en todas las comunidades autónomas, ni se exige en todos los municipios españoles. Como se ha expresado, la legislación estatal vigente obliga a que los municipios con población superior a 25.00o habitantes cuenten con una ordenanza específica que exija a los edificios de antigüedad superior a 50 años a contar con la ITE. En efecto, su implantación está determinada por el interés de los ayuntamientos en elaborar una ordenanza que regule las inspecciones y la recogida de datos. Para impulsar su desarrollo, la Federación Española de Municipios y Provincias (29) ofrece una ordenanza tipo reguladora de la ITE, en la que, para centralizar la recogida de datos, se incluye el registro informatizado del informe de evaluación tras la inspección realizada.
De acuerdo con los datos del Instituto Nacional de Estadística de 2011, en España existen alrededor de 300 municipios con una población superior a 25.00o habitantes que deberían contar con una ordenanza particular referente a la ITE. Sin embargo, según el Observatorio ITE promovido por el Ministerio de Fomento y desarrollado por el Instituto de la Construcción de Castilla y León, que realiza una encuesta anual a los municipios con una ordenanza específica de la ITE y con un registro de los informes, únicamente 89 municipios españoles tienen implantada dicha regulación y gestión (30). Sin embargo, esta información se considera insuficiente, dado que únicamente constan los municipios que han incorporado sus datos al Observatorio ITE. Por otro lado, algunos registros no están informatizados ni coordinados, ni tampoco georreferenciados.

Atendiendo al ámbito urbano, el Observatorio de la vulnerabilidad urbana del Ministerio de Fomento, es la entidad que analiza los datos relacionados con la vulnerabilidad urbana en España, en desarrollo de lo establecido en el citado texto refundido de la Ley de Suelo y Rehabilitación Urbana de 2015. Sin embargo, la última evaluación disponible en el Visor del Catálogo de Barrios Vulnerables en España (31) se realizó con datos de los Censos de Población y Vivienda, y del Padrón de habitantes, en las fechas de 1991, 2001, 2006, y 2011, contando con tres indicadores (paro, nivel de estudios y vivienda) y el indicador de inmigración para 2006 y 2011, todos ellos georreferenciados sobre las ciudades españolas mayores de 50.000 habitantes y las capitales de provincia, con lo cual es necesaria su actualización y desarrollo a nivel autonómico.

\section{OPORTUNIDADES QUE PRESENTAN LOS REGISTROS}

La existencia de un registro informatizado y georreferenciado de los datos sobre el estado de conservación de los edificios de viviendas en base a los informes IEE, así como de la vulnerabilidad urbana, permitiría la realización de diagnósticos eficientes para plantear políticas realistas de rehabilitación y regeneración urbanas.

El mencionado Plan Estatal de Vivienda 2018-2021, establece que, para el reconocimiento de las ayudas económicas previstas en el programa de fomento de la regeneración y renovación urbana y rural, la administración competente debe delimitar territorialmente el área de regeneración y renovación urbana o rural. Dicha delimitación debería hacerse con criterios prácticos, en base a la realidad del estado de conservación de los edificios y de la vulnerabilidad urbana del área en cuestión. La existencia de un registro geográficamente referenciado a escala urbana de los informes IEE, permitiría identificar las áreas necesitadas de regeneración y renovación con mayor exactitud, por reconocer en ellas los edificios en peor estado de conservación y situación de accesibilidad, y con peor eficiencia energética. Además, el registro de los informes IEE es clave dado que las actuaciones subvencionables según el Plan Estatal de Vivienda son, entre otras, la ejecución de obras de mantenimiento en edificios de viviendas para adecuarlos a los estándares de la normativa vigente, y la mejora de su eficiencia energética, cuestiones todas ellas incluidas en el IEE.

El actual Plan Estatal de Vivienda también establece como actuaciones subvencionables las obras de mejora de la ca- 
lidad y sostenibilidad del medio urbano, y previamente se exige un diagnóstico sobre la situación demográfica, social, económica y ambiental del área de regeneración y renovación urbana o rural. Por ello resulta fundamental contar con un registro georreferenciado de los indicadores que definen la vulnerabilidad urbana.

Por otro lado, el registro georreferenciado de los informes IEE y de la vulnerabilidad urbana crearía una interesante base de datos para su consulta en trabajos de investigación y proyectos de innovación. La información contenida en el IEE referente al estado de conservación, situación de accesibilidad y eficiencia energética de los edificios de viviendas, así como el registro del estado del espacio urbano puede ser de gran utilidad para el estudio de diversas temáticas. Según Gazulla, C. (32), es fundamental aplicar la visión del ciclo de vida, y tener en cuenta el comportamiento económico, ambiental y social de los edificios a la hora de identificar tecnologías, soluciones y productos más eficientes. En este sentido, la información registrada de los informes IEE referida a la fase de uso y mantenimiento de los edificios de viviendas, y el registro de la vulnerabilidad residencial a escala urbana resultaría de gran valor. Por otro lado, entre los indicadores que evalúan el comportamiento ambiental de los edificios, destaca el potencial de calentamiento global. Por ello las oportunidades de reducción del consumo de energía y las emisiones de $\mathrm{CO} 2$ en edificios existentes y áreas urbanas son objeto de múltiples investigaciones, siendo clave el conocimiento del comportamiento energético de los edificios a escala municipal. Asimismo, con vistas a analizar el comportamiento social de los edificios y las áreas urbanas, se incluyen aspectos tales como la accesibilidad, la adaptabilidad, la salud y el confort, y el mantenimiento y la seguridad (33), información toda ella contenida en el IEE y reflejada en los indicadores de la vulnerabilidad residencial de los espacios urbanos.

En relación a los edificios, es destacable la relevancia de la centralización del registro del IEE en organismos encargados de la tramitación, seguimiento y control de su contenido. De esta forma, a partir del IEE registrado, este sería validado, identificado y comunicado a la propiedad y al ayuntamiento. Los diferentes informes IEE realizados en un mismo edificio quedarían registrados, así como las posibles incidencias. De igual forma, sería recomendable elaborar un informe resumido de carácter público para que la información sobre el estado de conservación del parque edificado estuviera a disposición de los usuarios. Por su parte, las diferentes administraciones públicas deberían colaborar entre sí, de modo que la información de los informes IEE fuera accesible a los efectos de sus respectivas competencias. Así, cada ayuntamiento debería poder acceder al registro y extraer los informes de su municipio para velar por el deber de conservación de los edificios, exigiendo, en su caso, las subsanaciones correspondientes.

Atendiendo al espacio urbano, sería deseable la creación de entidades que, además de registrar y referenciar geográficamente en un mapa la vulnerabilidad urbana, gestionaran y actualizaran la información para llevar a cabo una evaluación fiable de las distintas zonas urbanas.

Es importante recalcar que los procedimientos de elaboración los informes de inspección técnica deberían desarrollarse con un procedimiento único para todo el territorio, para garantizar la homogeneidad de los contenidos en ese ámbito o, al menos, plantear tipos de ficheros que permitan intercambiar la información, independientemente del procedimiento con el que se elaboran. La realidad es que cada comunidad autónoma utiliza un procedimiento diferente con un enfoque y alcance distinto, hecho que dificulta un análisis a nivel territorial. El Plan de Vivienda 2013-2016 proponía, a nivel estatal, un anejo con un esquema y unos contenidos para los informes IEE, incluidos en una herramienta on line, pero que no ha sido utilizado por la comunidades que ya tenían implantados desde hace años procedimientos propios.

\section{EL CASO DEL REGISTRO DE EDIFICIOS EN LA COMUNITAT VALENCIANA}

Según lo expuesto, en 2007 se estableció la creación del Registro del Estado de Conservación de Edificios de Viviendas de la Comunitat Valenciana.

Desde entonces, los informes elaborados sobre edificios con edad superior a 50 años quedan incluidos, automáticamente, en dicho Registro a través de la correspondiente aplicación informática. Es importante resaltar que a los técnicos inspectores se les pide que recaben la información relativa al año de construcción real del edificio, para no confundirla con otras posteriores correspondientes a posibles reformas del mismo que recogen las bases de los catastros oficiales.

Según el Censo de Población y Viviendas del año 2011, elaborado por el Instituto Nacional de Estadística (INE), en la Comunidad Valenciana, existen más de 3.145.0oo viviendas, de las cuales, 850.00o tienen una antigüedad por encima de los 50 años, lo que supone casi un $60 \%$ del parque residencial. Consultando los datos del Registro, tan solo un $5 \%$ de las viviendas han sido inspeccionadas, lo que constata la escasa implantación del mismo.

A modo de ejemplo se expone información sobre el parque de sus edificios residenciales, a partir del análisis de los datos contenidos en 3.935 informes ICE registrados entre el 25 de noviembre de 2007 y el 30 de junio de 2011. A partir de los datos analizados, es posible afirmar que las intervenciones se concentraron en las fachadas y/o cubiertas de edificios de viviendas de tipología plurifamiliar entre medianeras de más de tres alturas. En relación a la tipología y antigüedad de los edificios, la mayoría de expedientes registrados correspondieron a edificios de vivienda colectiva excepto en la provincia de Castellón. Además, predominaron los expedientes resueltos en edificios entre medianeras de planta baja y tres alturas que databan del período entre 1961 y 1980, es decir, edificios que durante la inspección tenían entre 27 y 50 años. De este dato puede deducirse que la razón principal para realizar el informe ICE fue la solicitud de ayudas públicas para acometer actuaciones de rehabilitación.

Atendiendo a los elementos estructurales, a partir de los informes registrados puede conocerse tanto su estado de conservación como las tipologías inspeccionadas.

Así, se observa que el mayor porcentaje de expedientes que resultaron con peor estado de conservación de la estructura se correspondió con los forjados unidireccionales de madera y las vigas de madera (Tabla 1), aunque el número de edificios registrados con esta tipología estructural no fuera significativo (Figura 1). 
Tabla 1. Informes ICE registrados en la Comunitat Valenciana 2007-2011. Estado de diferentes tipos de estructura.

\begin{tabular}{|c|c|c|c|c|c|c|c|c|c|c|c|c|}
\hline \multirow[b]{2}{*}{ Tipos } & \multirow[b]{2}{*}{ Total } & \multicolumn{2}{|c|}{ Bueno } & \multicolumn{2}{|c|}{$\begin{array}{c}\text { Deficiencias } \\
\text { puntuales }\end{array}$} & \multicolumn{2}{|c|}{$\begin{array}{c}\text { Deficiencias } \\
\text { general }\end{array}$} & \multicolumn{2}{|c|}{ Malo } & \multicolumn{2}{|c|}{$\begin{array}{c}\text { Sin } \\
\text { determinar }\end{array}$} & \multirow{2}{*}{\begin{tabular}{|c|}
$\begin{array}{c}\text { Sin } \\
\text { estado }\end{array}$ \\
No \\
Exp
\end{tabular}} \\
\hline & & $\begin{array}{l}\text { No } \\
\text { Exp }\end{array}$ & $\%$ & $\begin{array}{c}\text { No } \\
\text { Exp }\end{array}$ & $\%$ & $\begin{array}{l}\text { No } \\
\text { Exp }\end{array}$ & $\%$ & $\begin{array}{l}\text { No } \\
\text { Exp }\end{array}$ & $\%$ & $\begin{array}{l}\text { No } \\
\text { Exp }\end{array}$ & $\%$ & \\
\hline Solera & 3.935 & 1.171 & 29,76 & 495 & 12,58 & 187 & 4,75 & 46 & 1,17 & 1.140 & 28,97 & 896 \\
\hline Muro de carga/Fábrica & 1.912 & 808 & 42,26 & 681 & 35,62 & 229 & 11,98 & 43 & 2,25 & 151 & 7,90 & \\
\hline Muro de carga/Hormigón & 97 & 52 & 53,61 & 24 & 24,74 & 6 & 6,19 & 2 & 2,06 & 13 & 13,40 & \\
\hline Pilares/Metálicos & 210 & 135 & 64,29 & 25 & 11,90 & 6 & 2,86 & $\mathrm{O}$ & 0,00 & 44 & 20,95 & \\
\hline Pilares/ Hormigón & 2.875 & 1.930 & 67,13 & 376 & 13,08 & 89 & 3,10 & 79 & 2,75 & 401 & 13,95 & \\
\hline Pilares/Madera & 18 & 7 & 38,89 & 2 & 11,11 & 7 & 38,89 & $\mathrm{o}$ & 0,00 & 2 & 11,11 & \\
\hline Pilares/Fábrica & 883 & 534 & 60,48 & 208 & 23,56 & 37 & 4,19 & 19 & 2,15 & 85 & 9,63 & \\
\hline Vigas/Metálicas & 593 & 347 & 58,52 & 123 & 20,74 & 35 & 5,90 & 13 & 2,19 & 75 & 12,65 & \\
\hline Vigas/ Hormigón & 1.645 & 1.067 & 64,86 & 268 & 16,29 & 48 & 2,92 & 41 & 2,49 & 221 & 13,43 & \\
\hline Vigas/ Madera & 1.212 & 323 & 26,65 & 354 & 29,21 & 209 & 17,24 & 177 & 14,60 & 149 & 12,29 & \\
\hline Forjado/Unidireccional/Hormigón & 2.854 & 1.440 & 50,46 & 724 & 25,37 & 179 & 6,27 & 122 & 4,27 & 389 & 13,63 & \\
\hline Forjado/Unidireccional/Madera & 1.432 & 386 & 26,96 & 435 & 30,38 & 278 & 19,41 & 205 & 14,32 & 128 & 8,94 & \\
\hline Forjado/Unidireccional/Cerámico & 405 & 150 & 37,04 & 127 & 31,36 & 37 & 9,14 & 33 & 8,15 & 58 & 14,32 & \\
\hline Forjado/Reticular & 40 & 27 & 67,50 & 9 & 22,50 & 1 & 2,50 & 1 & 2,50 & 2 & 5,00 & \\
\hline Forjado/Losa & 79 & 35 & 44,30 & 19 & 24,05 & 11 & 13,92 & 8 & 10,13 & 6 & 7,59 & \\
\hline
\end{tabular}

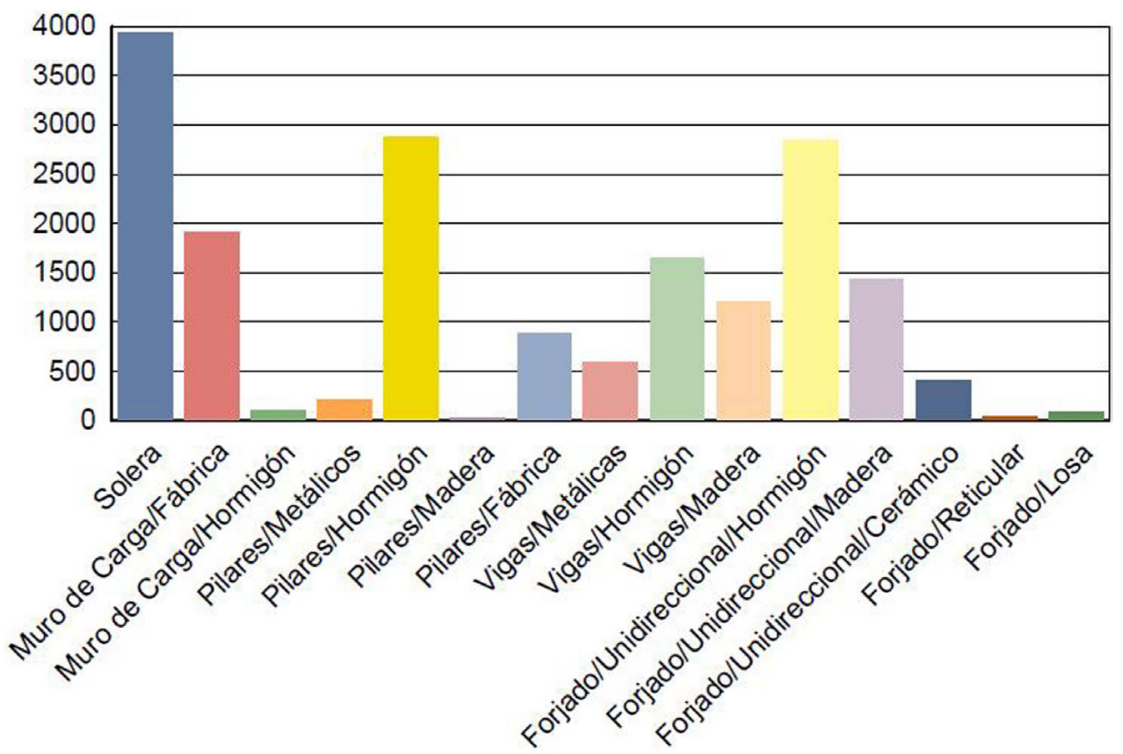

Figura 1. Informes ICE registrados en la Comunitat Valenciana 2007-2011.

Número de expedientes por tipos de estructura.

En referencia a las fachadas, se constata que los revestimientos con aplacado de piedra y alicatado cerámico comportaron peor estado de conservación. Sin embargo, este tipo de revestimientos tuvo muy poca relevancia en los expedientes analizados. Por último, se comprueba que el mayor porcentaje de informes que presentaron peor estado de conservación de cubiertas se dio en ciertos componentes de cubiertas inclinadas, tales como la impermeabilización, los elementos singulares y la recogida de aguas. Además, estos elementos existían en tipos de cubiertas inclinadas que se encontraron con bastante frecuencia en el período evaluado.

En lo referente a la accesibilidad, en la mayor parte de los informes registrados se propuso algún tipo de intervención, destacando la colocación de ascensor con supresión de barreras, para satisfacer la accesibilidad universal en el conjunto del edificio. La ubicación propuesta para el ascensor fue, en gran parte de los casos, el hueco de escalera, y en muy pocos expedientes se planteó la solución por fachada exterior.
Según lo comentado anteriormente, la georreferenciación a escala urbana de datos referentes al estado de conservación de los edificios supondría múltiples oportunidades para las distintas administraciones y el desarrollo de proyectos de investigación.

En la Comunitat Valenciana, la inclusión de los informes IEEV.CV registrados en el visor cartográfico de la Generalitat Valenciana (34) constituye un ejemplo de georreferenciación. Como dispone el ya mencionado Decreto 53/2018, los informes IEEV.CV se inscriben de forma telemática en el registro autonómico. Este registro identifica cada edificio por su referencia catastral, de forma que los datos del IEEV.CV sirven para documentar los censos sobre necesidades de rehabilitación de viviendas en la Comunitat Valenciana. Para dar más agilidad y visibilidad a este proceso, el centro directivo que gestiona el registro autonómico del IEEV.CV en la Comunitat Valenciana, en colaboración con el Instituto Cartográfico Valenciano, ha incorporado en el visor cartográfico de la Gene- 
ralitat Valenciana la identificación en un mapa de la Comunitat de todos los edificios de más de 50 años, en color rojo, y de los edificios de uso residencial con IEEV.CV, en color verde (Figura 2). De esta forma se pueden reconocer aquellos edificios cuyos propietarios tienen pendiente la promoción del informe IEEV.CV. Además, la información georreferenciada en el visor se actualiza de forma inmediata pues lee directamente del registro de informes IEEV.CV.

El visor cartográfico ofrece información resumida del informe IEEV.CV accesible a cualquier persona usuaria. Si se precisa información más detallada sobre el estado de conservación por parte de personal investigador, es posible conseguirla a través del registro autonómico del IEEV.CV. Por su parte, cada ayuntamiento puede acceder y descargar del registro los informes correspondientes a los edificios de su municipio, para realizar el control y seguimiento sobre la obligación del deber de conservación y rehabilitación de estos.

Otro ejemplo interesante de registro y georreferenciación de datos de edificios es la herramienta informática ENERFUND (35), resultado de un proyecto internacional con 15 organismos socios, entre los que figura el Instituto Valenciano de la Edificación, y que ha sido financiado dentro del programa
Horizonte 2020 de la Unión Europea. La herramienta online ENERFUND ofrece los resultados del certificado de eficiencia energética de edificios de forma georreferenciada a escala nacional de los países de origen de los socios del proyecto. De esta forma es posible identificar gráficamente en un mapa el comportamiento energético de las distintas zonas edificadas (Figura 3). Igualmente, esta aplicación permitirá calificar las oportunidades de rehabilitación energética a partir de parámetros tales como el certificado de eficiencia energética mencionado, el número de instaladores certificados, los programas gubernamentales vigentes, etc. En base a la calificación ofrecida por ENERFUND, las empresas de servicios o productos podrán identificar tipos de clientes según sus necesidades y las administraciones competentes podrán evaluar, comparar edificios y decidir sobre la asignación de fondos públicos para su rehabilitación energética.

Es importante recalcar el gran trabajo de depuración de datos contenidos en los informes de certificación energética que ha tenido que desarrollarse. Se han detectado certificaciones duplicadas, algunas estaban ubicadas en otras comunidades, algunos de los valores de consumos energéticos eran dudosos, .... El origen de este problema podría estar en que son registros de reciente creación, con procedimientos



Figura 2. Pantalla del visor cartográfico de la Generalitat Valenciana (https://visor.gva.es/visor/). Informe de evaluación del edificio (centro de Valencia).

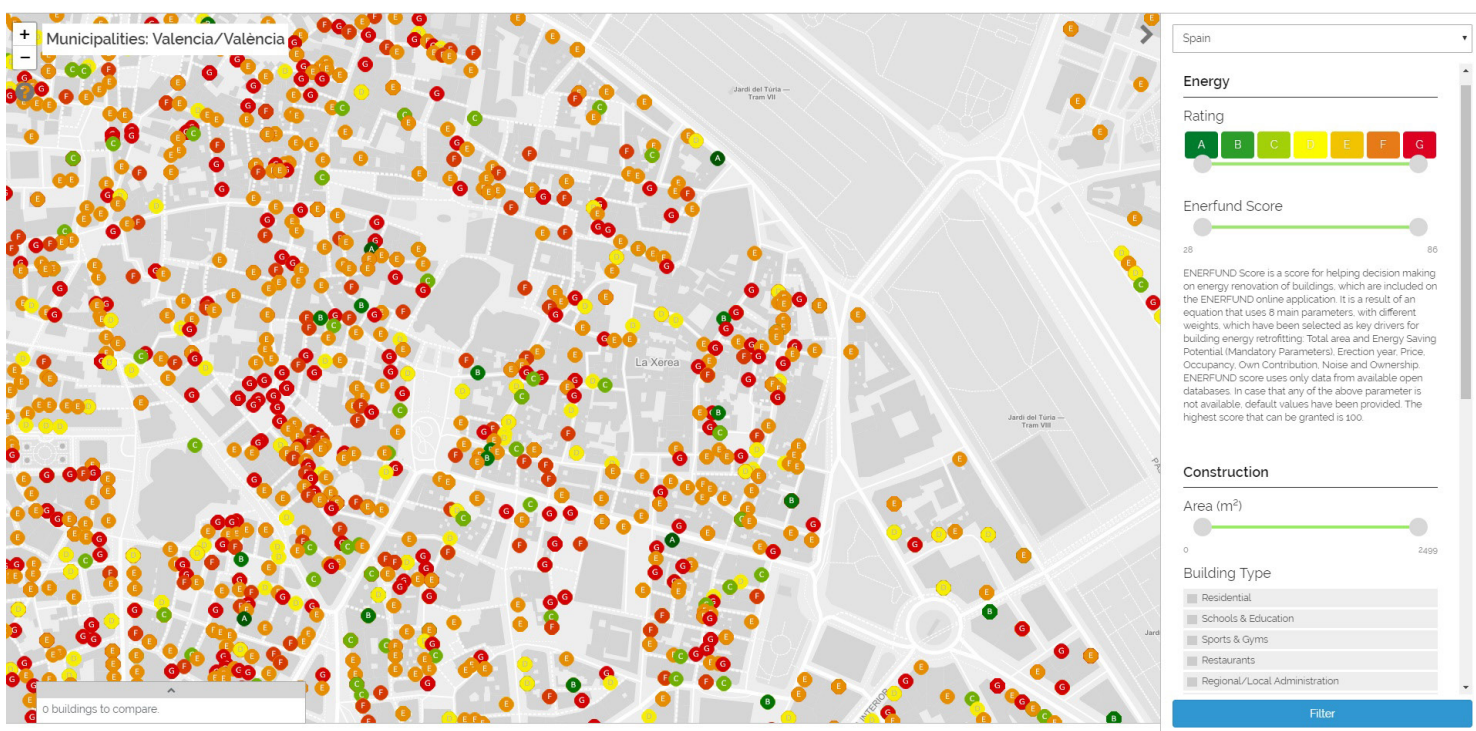

Figura 3. Pantalla de la herramienta informática ENERFUND (http://app.enerfund.eu/) (centro de Valencia). 
que han sufrido cambios de mejora continua, pasando de un sistema manual, sin mecanismos que permitan validar los datos y contrastar la información, a un registro automatizado, controlado y sistemático. Por ello, se han tenido que adoptar medidas para depurar la base de datos inicial con casi 400.000 registros. Por ejemplo, en el caso de existencia de más de una certificación sobre un edificio de viviendas se ha seleccionado aquel que contiene la información del edificio completo, frente a los que tiene certificados de viviendas aisladas y, los de menor antigüedad. Se han tenido que descartar valores aberrantes o ubicaciones que no procedían. Finalmente, se ha conseguido trabajar con una base de datos de unos 350.00o registros. La georreferenciación de la base de datos con sistemas GIS ha facilitado la detección de errores y ha ayudado a inferir calidad y fiabilidad al registro de certificados.

En la Comunitat Valenciana, también se ha elaborado el Visor de Espacios Urbanos Sensibles, VEUS. El objeto de VEUS es la identificación cartográfica de las áreas urbanas sensibles, entendiendo como tales las unidades censales donde las tres dimensiones que definen la vulnerabilidad: socio- económica, residencial y socio-demográfica, son menores que la media de la Comunitat. VEUS funciona a partir de datos estadísticos, con la particularidad de que estos son cotejados por las administraciones locales a través de un cuestionario específico, dando lugar a un proceso muy participativo. Así, resulta posible calificar cada dimensión de la vulnerabilidad según una combinación de nueve variables de análisis. Por ejemplo, la vulnerabilidad residencial (Figura 4) se determina a partir de datos relativos a la antigüedad de la edificación, el valor catastral y la accesibilidad, pudiendo resultar vulnerabilidad residencial alta, (identificada en color rojo), media, (en color amarillo) o baja, (en color verde).

A partir de la calificación de las dimensiones se determina el grado de vulnerabilidad, que puede ser integral (tres de las dimensiones alcanzan nivel alto), polivulnerabilidad (dos de las dimensiones tienen nivel alto o existen dos dimensiones con nivel medio y alto respectivamente), o bien vulnerabilidad residual (resto de combinaciones posibles).
El visor cartográfico de la Generalitat Valenciana también incorpora los espacios urbanos sensibles (EUS), definidos como agrupaciones de secciones censales en las que están presentes niveles de vulnerabilidad integral o polivulnerabilidad, y con una puntuación de las variables superior a un determinado valor.

Finalmente, en cada sección censal de VEUS es posible visualizar una ficha resumen que indica si se trata de un espacio urbano sensible o no, y ofrece la calificación de la vulnerabilidad socio económica, residencial y socio demográfica, el factor corrector por población, la tipología de vulnerabilidad activada en el visor, la puntuación total de la vulnerabilidad, así como el detalle del valor y la tipificación de las nueve variables de análisis.

Toda esta información sobre el estado de conservación de los edificios a nivel de secciones censales es necesaria para que las distintas administraciones desarrollen políticas concretas y establezcan prioridades adaptadas a la realidad actual en materia de rehabilitación y regeneración urbana. Como ya se ha explicado, el programa de fomento de la regeneración y renovación urbana y rural del vigente Plan Estatal de Vivienda 2018-2021 establece una línea de financiación a los ayuntamientos gestionada por las comunidades autónomas. El objeto de este programa es la realización conjunta de obras de rehabilitación en edificios y viviendas, incluidas viviendas unifamiliares, obras de urbanización o reurbanización de espacios públicos y, en su caso, obras de edificación en sustitución de edificios o viviendas demolidos dentro de ámbitos de actuación denominados áreas de regeneración y renovación urbana o rural previamente delimitados. El desarrollo del Plan Estatal de Vivienda en la Comunitat Valenciana, establece que en la concesión de ayudas económicas a las áreas de regeneración urbana y rural (ARRUR) se puntuará la necesidad de intervención en base al grado de vulnerabilidad del ámbito de intervención según VEUS, dando prioridad a los espacios urbanos sensibles. Asimismo, los edificios que requieran intervención dentro del ámbito delimitado deberán contar con el correspondiente informe IEEV.CV, que puede consultarse en el visor cartográfico de la Generalitat Valenciana, como



Figura 4. Pantalla del visor cartográfico de la Generalitat Valenciana (https://visor.gva.es/visor/). Visor de Espacios Urbanos Sensibles - VEUS. Vulnerabilidad residencial (Comunitat Valenciana). 
ya se ha comentado. En el visor cartográfico se incorporan las ARRUR con una ficha resumen de consulta pública que recoge datos generales, análisis y necesidades, descripción de la intervención, costes, ayudas públicas, delimitación del área, análisis y diagnóstico, fotografías, objetivos estratégicos y actuaciones.

Por otro lado, el visor cartográfico de la Generalitat Valenciana también incluye la Estrategia de Desarrollo Urbano Sostenible e Integrado (EDUSI), que identifica los municipios con financiación aprobada según las tres convocatorias propuestas a partir del Reglamento FEDER (36) el cual establece ayudas económicas para que los organismos subregionales o locales lleven a cabo actuaciones de desarrollo urbano sostenible en determinadas zonas como contribución a los objetivos de la Estrategia Europa 2020. En el visor cartográfico es posible descargar una ficha para cada municipio que resume la siguiente información: datos del municipio, tramitación del EDUSI, descripción de la estrategia (delimitación del área, líneas de actuación, objetivos), necesidad de intervención, plan financiero y análisis DAFO (debilidades, amenazas, fortalezas y oportunidades).

Además, el visor cartográfico de la Generalitat Valenciana es pionero en España en incorporar, de forma georreferenciada, precios del alquiler privado de vivienda.

Estos precios han sido calculados a partir de los datos del Registro de Fianzas de arrendamiento de la Comunitat Valenciana de los tres últimos años, pasando un proceso de depuración hasta dejar constancia de los edificios cuya superficie construida destinada a vivienda es superior al 50\%. En este caso el mapa se divide en agrupaciones de zonas que tienen un comportamiento similar y donde existe un mínimo de 50 datos disponibles del registro de fianzas, resultando diez zonas en la Comunitat (Figura 5). Cada una de las zonas cuenta con una ficha que incorpora, para tres rangos de superficie de viviendas (40-75 m2, 76-100 m2 y 101-200 m2) y dos periodos de construcción si se considera relevante (hasta 1980 y a partir de 1981), tres precios del alquiler privado de vivienda en euros por m2 (valor límite inferior, valor medio y valor límite superior). Estos valores de alquiler representan un in- dicador económico real sobre la evolución del mercado y regulan los precios del alquiler de vivienda a nivel orientativo.

Toda la información anteriormente expuesta es fuente de datos para el Observatorio del Hábitat y Segregación Urbana de la Comunitat Valenciana (OHSU) (37) que ha sido creado para centralizar y conocer la situación de la vivienda a partir de una información cuantitativa, cualitativa y su distribución territorial permanentemente actualizada, y así poder conducir los intereses de la sociedad a la Administración, y en su caso, proponer las medidas necesarias. De esta forma, este observatorio participará en la evaluación y diseño de las políticas públicas en materia de vivienda e integración urbana a partir del diagnóstico y seguimiento de la situación habitacional de las personas, los recursos de habitabilidad públicos y privados, y la elaboración de un sistema de indicadores que identifiquen las áreas que presenten segregación urbana. Concretamente, es el organismo responsable de establecer el precio de referencia de alquiler en la Comunitat Valenciana, así como las zonas tensionadas en las que se han proudcido incrementos de precios desproporcionados. Los propietarios que se acojan a este valor, en dichas zonas, obtendrán beneficios fiscales, como medida para prevenir la creación de una burbuja del alquiler.

\section{CONCLUSIONES}

En los últimos años la realización del informe IEE se ha visto impulsada por el gran avance normativo en materia de rehabilitación. Las ayudas económicas para su redacción, así como la condición previa de contar con el IEE para otorgar ayudas económicas a la rehabilitación han favorecido su implantación. En consecuencia, el IEE se ha convertido en un documento que informa y conciencia a los propietarios sobre la necesidad de realizar intervenciones correctivas en el edificio.

En la Comunitat Valenciana el contenido del IEE también es una referencia para la confección, por voluntad de las comunidades de propietarios, del programa de mantenimiento que forma parte del Libro del Edificio rehabilitado, lo cual supone una ayuda para fomentar la cultura del mantenimiento en los edificios.



Figura 5. Pantalla del visor cartográfico de la Generalitat Valenciana (https://visor.gva.es/visor/). Precio del alquiler privado en la Comunitat Valenciana. 
Asimismo, ha evolucionado la regulación sobre la obligatoriedad de constituir registros con los resultados del IEE, aunque su implantación a nivel nacional es todavía incipiente, por lo que sigue existiendo una carencia de datos sobre el estado de conservación del parque edificatorio residencial en España, información muy necesaria por la avanzada edad de este.

El registro de datos de los informes IEE en cada comunidad autónoma debería estar centralizado, informatizado y se debería poder consultar de forma pública. De este modo los propietarios podrían conocer la problemática de sus inmuebles, incentivo importante para acometer las intervenciones necesarias.

Si los datos de los informes IEE estuvieran, además, georreferenciados a escala urbana, las distintas administraciones podrían realizar diagnósticos eficientes para desarrollar políticas realistas de rehabilitación y regeneración urbana.

Por otro parte, se debería impulsar el desarrollo de aplicaciones informáticas que registren e identifiquen cartográficamente el estado del espacio urbano a nivel de sección censal, de forma que las diferentes administraciones adaptaran a la realidad sus políticas de ayudas a la rehabilitación edificatoria y la regeneración y renovación urbanas. De hecho, la actual normativa ya exige una evaluación previa de las zonas urbanas para acceder a la financiación oficial de las actuaciones, con lo cual se hace más necesaria la georreferenciación de los indicadores que definen la vulnerabilidad urbana.

Toda esta información georreferenciada serviría como provisión de datos para los observatorios de la vivienda, organismos cuya creación debería fomentarse a nivel autonómico para centralizar la información sobre unidades administrativas comunes en el ámbito estatal.
Además, es de destacar que el contenido del IEE y los indicadores que definen el estado del espacio urbano, representan una valiosa fuente de información para el desarrollo de trabajos de investigación y proyectos de innovación, por lo que la constitución de registros con esta información y el acceso público a estos supondría una ayuda de gran utilidad en la actividad I+D+i.

En definitiva, el conocimiento en profundidad del estado del parque de edificios existentes y del espacio urbano resulta absolutamente imprescindible como diagnóstico previo para impulsar la rehabilitación dentro del sector de la construcción y ayudar a su reactivación atendiendo a las necesidades reales de las personas.

Por último, para conseguir realmente todas las posibles ventajas que se plantean en el presente documento relativas a tener constituidos registros oficiales con información del parque residencial existente, es imprescindible, por una lado, garantizar la calidad y fiabilidad de la información registrada a través de procesos sistemáticos e inteligentes de entrada de datos y, por otro, plantear procedimientos homogéneos para distintas comunidades o, al menos, que permitan el trabajo con ficheros de intercambio de información.

\section{AGRADECIMIENTOS}

Isabel de los Rios Ruipérez por la validación de la información relativa a la normativa urbanística y sobre el Informe de Evaluación de Edificios (IEE).

Alberto Sanchis Cuesta por la información facilitada sobre los resultados del registro de Informe de Evaluación de Edificios (IEE) en la Comunidad Valenciana.

Al Instituto Valenciano de la Edificación por toda la documentación facilitada sobre visores de edificios y espacios urbanos.

\section{REFERENCIAS}

(1) Dirección General de Arquitectura, Vivienda y Suelo; Ministerio de Fomento (2014). Observatorio de Vivienda y Suelo. Boletín Especial Censo 2011 Parque edificatorio. España. Recuperado de https://www.fomento.gob.es/MFOM. CP.Web/handlers/pdfhandler.ashx?idpub=BAW021

(2) Ministerio de Gracia y Justicia (1889). Real Decreto de 24 de julio de 1889 por el que se publica el Código Civil. Boletín Oficial del Estado, n ${ }^{\circ}$ 206. España.

(3) Jefatura del Estado (1956). Ley de 12 de mayo de 1956 sobre Régimen del Suelo y Ordenación Urbana. Boletín Oficial del Estado, $\mathrm{n}^{\mathrm{o}}$ 135. España.

(4) Ministerio de la Vivienda (1976). Real Decreto 1346/1976, de 9 de abril, por el que se aprueba el Texto Refundido de la Ley sobre Régimen del Suelo y Ordenación Urbana. Boletín Oficial del Estado, nº 144 . España.

(5) Jefatura del Estado (1990). Ley 8/1990, de 25 de julio sobre Reforma del Régimen Urbanístico y Valoraciones del Suelo. Boletín Oficial del Estado, no 17 . España.

(6) Ministro de Obras Públicas y Transportes (1992). Real Decreto Legislativo 1/1992, de 26 de junio, por el que se aprueba el Texto Refundido de la Ley sobre el Régimen del Suelo y Ordenación Urbana. Boletín Oficial del Estado, $n^{\circ} 156$. España.

(7) Jefatura de Estado (1998). Ley 6/1998, de 13 de abril, sobre régimen del suelo y valoraciones. Boletín Oficial del Estado, no 89. España.

(8) Jefatura del Estado (1999). Ley 38/1999, de 5 de noviembre, de Ordenación de la Edificación. Boletín Oficial del Estado, $n^{0}$ 266. España.

(9) Jefatura del Estado (2007). Ley 8/2007, de 28 de mayo, de suelo. Boletín Oficial del Estado, no ${ }^{0}$ 128. España.

(10) Ministerio de Vivienda (2008). Real Decreto Legislativo 2/2008, de 20 de junio, por el que se aprueba el texto refundido de la Ley de Suelo. Boletín Oficial del Estado, no ${ }^{154}$. España.

(11) Ministerio de Fomento (2015). Real Decreto Legislativo 7/2015, de 30 de octubre, por el que se aprueba el texto refundido de la Ley de Suelo y Rehabilitación Urbana. Boletín Oficial del Estado, no 261 . España. 
(12) Jefatura del Estado (2011). Real Decreto-ley 8/2011, de 1 de julio, de medidas de apoyo a los deudores hipotecarios, de control del gasto público y cancelación de deudas con empresas y autónomos contraídas por las entidades locales, de fomento de la actividad empresarial e impulso de la rehabilitación y de simplificación administrativa. Boletín Oficial del Estado, no 161 . España.

(13) Jefatura del Estado (2011). Ley 2/2011, de 4 de marzo, de Economía Sostenible. Boletín Oficial del Estado, nº 55. España.

(14) Ministerio de Fomento (2013). Real Decreto 233/2013, de 5 de abril, por el que se regula el Plan Estatal de fomento del alquiler de viviendas, la rehabilitación edificatoria, y la regeneración y renovación urbanas, 2013-2016. Boletín Oficial del Estado, no 86. España.

(15) Ministerio de Vivienda (2006). Real Decreto 314/2006, de 17 de marzo, por el que se aprueba el Código Técnico de la Edificación. Boletín Oficial del Estado, $\mathrm{n}^{0}$ 74. España.

(16) Ministerio de la Presidencia (2013). Real Decreto 235/2013, de 5 de abril, por el que se aprueba el procedimiento básico para la certificación de la eficiencia energética de los edificios. Boletín Oficial del Estado, no 89. España.

(17) Jefatura del Estado (2013). Ley 8/2013, de 26 de junio, de rehabilitación, regeneración y renovación urbanas. Boletín Oficial del Estado, no 153 . España.

(18) Tribunal Constitucional (2017). Pleno. Sentencia 143/2017, de 14 de diciembre de 2017. Recurso de inconstitucionalidad 5493-2013. Boletín Oficial del Estado, nº 15. España.

(19) Presidencia de la Generalitat (2019). Ley 1/2019, de 5 de febrero, de la Generalitat, de modificación de la Ley 5/2014, de 25 de julio, de ordenación del territorio, urbanismo y paisaje de la Comunitat Valenciana. Diario Oficial de la Comunitat Valenciana, no 8481. España.

(20) Conselleria de Infraestructuras, Territorio y Medio Ambiente (2014). Resolución de 8 de septiembre de 2014, de la Dirección General de Obras Públicas, Proyectos Urbanos y Vivienda, relativa a la implementación en la Comunitat Valenciana del informe de evaluación del edificio a partir del informe de conservación y de la certificación energética del edificio. Diario Oficial de la Comunitat Valenciana, $\mathrm{n}^{0}$ 7374. España.

(21) Conselleria de Infraestructuras, Territorio y Medio Ambiente (2015). Orden 7/2015, de 24 de marzo, por la que se aprueban las bases que reguladoras del Programa de Apoyo a la Implantación del Informe de Evaluación de los Edificios para el periodo 2013-2016, y se convocan las ayudas para el ejercicio 2015. Diario Oficial de la Comunitat Valenciana, ${ }^{\circ}$ 7496. España.

(22) Conselleria de Vivienda, Obras Públicas y Vertebración del Territorio (2016). Orden 5/2016, de 19 de mayo, de la Conselleria de Vivienda, Obras Públicas y Vertebración del Territorio, por la que se aprueban las bases reguladoras del Programa de fomento de la rehabilitación edificatoria y se convocan las ayudas para el ejercicio 2016. Diario Oficial de la Comunitat Valenciana, $\mathrm{n}^{0}$ 7790. España.

(23) Ministerio de Fomento (2015). Real Decreto Legislativo 7/2015, de 30 de octubre, por el que se aprueba el texto refundido de la Ley de Suelo y Rehabilitación Urbana. Boletín Oficial del Estado, n⿳0 261. España.

(24) Conselleria de Vivienda, Obras Públicas y Vertebración del Territorio (2018). Decreto 53/2018, de 27 de abril, del Consell, por el que se regula la realización del informe de evaluación del edificio de uso residencial de vivienda y su Registro autonómico en el ámbito de la Comunitat Valenciana. Diario Oficial de la Comunitat Valenciana, no 8288. España.

(25) Conselleria de Vivienda, Obras Públicas y Vertebración del Territorio, Instituto Valenciano de la Edificación (2018). España. Aplicación informática IEEV.CV. Recuperado de http://www.habitatge.gva.es/es/web/arquitectura/aplicacioninforme-de-evaluacion-del-edificio

(26) Conselleria de Vivienda, Obras Públicas y Vertebración del Territorio, Instituto Valenciano de la Edificación, Universidad Politécnica de Valencia, Asociación Técnica Española de Climatización y Refrigeración (2017). Método Abreviado CERMA v 4.2.5. Recuperado de https://energia.gob.es/desarrollo/EficienciaEnergetica/CertificacionEnergetica/DocumentosReconocidos/Paginas/procedimientos-certificacion-proyecto-terminados.aspx

(27) Conselleria de Vivienda, Obras Públicas y Vertebración del Territorio, Instituto Valenciano de la Edificación (2018). Programa de Operaciones de Mantenimiento de Edificios Existentes (POMEES). Recuperado de http://www.five.es/ productos/herramientas-on-line/pomees/

(28) Ministerio de Fomento (2018). Real Decreto 106/2018, de 9 de marzo, por el que se regula el Plan Estatal de Vivienda 2018-2021. Boletín Oficial del Estado, n⿳0 61. España.

(29) Federación Española de Municipios y Provincias (2013). Ordenanza tipo reguladora del Informe de Evaluación de los Edificios. Recuperado de http://femp.femp.es/Microsites/Front/PaginasLayout2/Layout2_Personalizables/MS_ Maestra_2/_k6sjJ7QfK2bAXnzzwDKDBpl7gtxuuvKz2xE6jhF1xawPpcqspDM6tpeczuXnxG6o

(30) Observatorio ITE (2018). Inspección técnica de edificios iteweb.es. Mapa ITE. Recuperado de http://www.iteweb.es/

(31) Ministerio de Fomento (2018). Visor del Catálogo de Barrios Vulnerables. Recuperado de https://apps.fomento.gob.es/ barriosvulnerables

(32) Construction 21 (2018). Análisis del Ciclo de Vida de productos y edificios. Gazulla, C. Recuperado de https://www. construction21.org/espana/articles/es/analisis-del-ciclo-de-vida-de-productos-y-edificios.html

(33) AENOR-CEN (2012). UNE-EN 15643-3. Sostenibilidad en la construcción. Evaluación de la sostenibilidad de los edificios. Parte 3: Marco para la evaluación del comportamiento social. Asociación Española de Normalización (AENOR).

(34) Conselleria de Vivienda, Obras Públicas y Vertebración del Territorio. Instituto Cartográfico Valenciano (2018). Visor cartográfico de la Generalitat Valenciana. Recuperado de https://visor.gva.es/visor/

(35) ENERFUND (2018) Recuperado de http://app.enerfund.eu/

(36) Unión Europea (2013). Reglamento (UE) nº 1301/2013 del Parlamento Europeo y del Consejo, de 17 de diciembre de 2013, sobre el Fondo Europeo de Desarrollo Regional y sobre disposiciones específicas relativas al objetivo de inversión en crecimiento y empleo y por el que se deroga el Reglamento (CE) nº 1080/2006. Diario Oficial de la Unión Europea, L 347. 
(37) Conselleria de Vivienda, Obras Públicas y Vertebración del Territorio (2018). Decreto 9/2019, de 1 de febrero, del Consell, de creación del Observatorio del Hábitat y Segregación Urbana de la Comunitat Valenciana. Diario Oficial de la Comunitat Valenciana, no 8480 . España. 